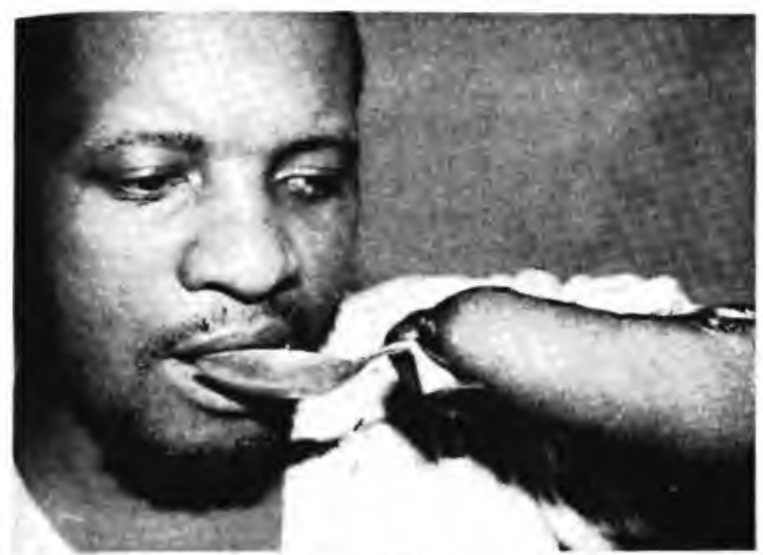

Fig. 3. Eating

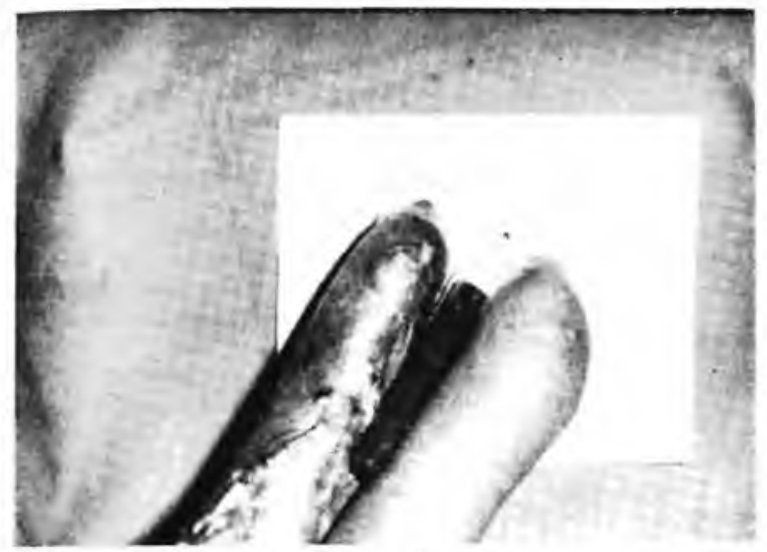

Fig. 4 Writing

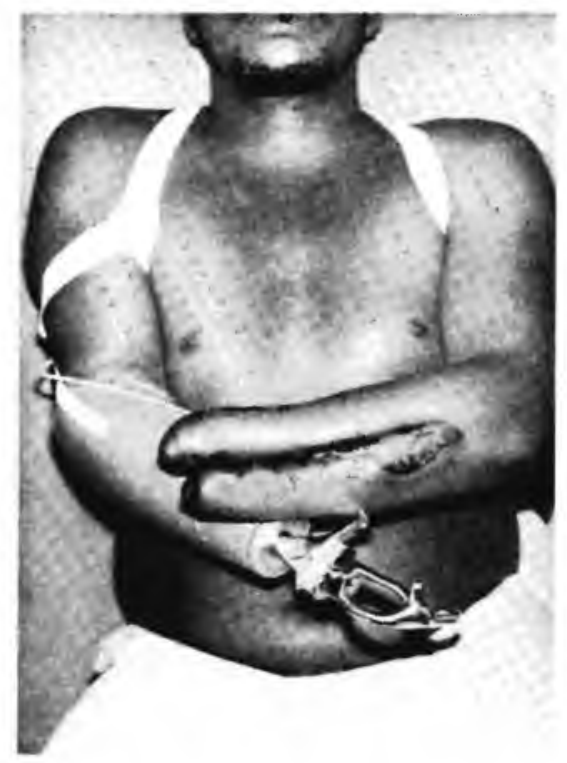

Fig. 4. Final State.

\section{Would You wear a Prosthesis?}

\author{
MARJORIE F. HUMPHREY, \\ B.Sc. Phy. (Rand), Dip. Prosthetics (N.Y.U.)
}

Skeletons in a cupboard? How many, when taken out, unurapped and carefully dusted down, are likely to turn out to be two stately legs, one dejected shoulder prosthesis and a miscellany of upper and lower limb misfits? Each representing a degree of heartbreak, frustration, expense and time wasted by a considerable number of people?

The reasons for rejection are many but could be overcome to some extent if, before prescribing a prosthesis, sufficient thought were given to the following: age, sex, I.Q. and type of patient, comfort, time lag, suitability of prosthesis ordered, availability of repair services and cost.

A child who has been born without limbs will readily accept an appliance for upper or lower limbs if it is comfortable and will give him a measure of independence. He will learn to manipulate fairly complicated apparatus, provided he finds it functional, but will discard anything which is uncomfortable and where the effort required to perform a task is out of proportion to the result achieved.

A young man or woman who is otherwise physically fit, will usually accept one or two lower limb prostheses whereas the elderly will reject any appliance which requires too much effort and puts a strain on their physical and mental resources.

Next to the bi-lateral above knee prosthesis, the one most likely to be rejected is the above elbow prosthesis. As the A.E. stump gets shorter the likelihood of rejection becomes greater. The converse applies in the below elbow prosthesis. The longer the stump, the more likely the patient is liable to discard a mechanical device in favour of a surface which will provide him with a sense of touch.

In bi-lateral A.E. amputations, men and women in both age groups will usually wear at least one appliance - usually on the dominant side. In unilateral amputees, young men are more likely to wear a functional appliance than women. Women will wear a cosmetic arm more often than men. If the dominant hand remains, the chances of rejection are greater than if the dominant hand is lost. Elderly men and women will usually reject an appliance on the grounds of discomfort and effort required to manipulate the appliance. It should be borne in mind that, with a functional prosthesis, the harness is apt to restrict movement of the sound arm.

In a sophisticated society more amputees will wear either a functional or cosmetic appliance, whereas the less sophisticated will only wear an appliance if it is useful. It will only be useful if it is simple, sturdy, with movable and removable parts reduced to a minimum and able to withstand being attacked with a hammer or rock to "improve" function. All groups are bound to fall victim to the vast mass of "compulsive fiddlers", that happy band who have only to see something mechanical to fall upon it to "fix", "adjust" and "set", regardless of the fact that they have probably failed their basic grade in Physical Science!

The cost of most prostheses is exorbitant, therefore it is the patient who has had to pay for the limb himself who is most likely to persevere and derive some benefit from his purchase.

Perhaps the greatest reason for rejection is the delay or time lag between amputation and fitting of the limb. In traumatic amputations other injuries may demand priority and in the ensuing delay the patient becomes used to managing without $a \operatorname{limb}$ and is often not prepared to persevere in the use of an appliance which he feels he can well do without. Elective surgery is usually performed because of disease or deformity. In the case of disease, the patient's general condition is below par and he tends to discard the prosthesis for the least excuse. Amputation because of deformity is much more successful, particularly if a weight-bearing stump can be preserved and a simple protective or cosmetic appliance is all that is required. 